Hence for sufficiently large $k \in K$, from (4.11) we know $w_{J_{+} \cap Q^{k}}^{k}=0$. On the other hand, from the fact $\left|J_{+} \cap Q^{*}\right|=\left|Q_{+}^{*}\right|$ and (4.11) we have $\left|J_{+} \cap Q^{k}\right|=\left|Q_{+}^{k}\right|$, hence, from the definition of $\Omega_{k}$, we easily obtain $\Omega_{k}=\emptyset$ for sufficiently large $k \in K$. Thus $s^{k}(k \in K)$ are all defined by $\langle 14\rangle$, which contradicts the fact that $s^{k}$ is all defined by $\langle 10\rangle$ for any $k \in K$. Hence (4.7) is true and the lemma follows.

We conclude this paper by proving the following theorem.

Theorem 4.3. If (H2), (H3) and (H4) are satisfied, then the infinite point sequence $\left\{x^{k}\right\}$ generated by our algorithm converges superlinearly to the unique optimal solution $x^{*}$ of (NP).

Proof. From (4.11) and Lemma 4.2, $Q^{k}$ is equal to some fixed $Q$ for sufficiently large $k$. The algorithm in Section 3 is equivalent to the variable metric algorithm described in [8] on the hyperplane $\left\{x \mid a_{j}^{T} x=b_{j}(j \in Q)\right\}$. According to the results of [8], we obtain the conclusion of our theorem.

\title{
References
}

[1] D. Goldfarb. Extension of Davidon's Variable Metric Method to Maximization under Linear Inequality and Equality Constraints. SIAM J. Applied Math., 1969, 17: 739-764.

[2] J. Rosen. The Gradient Projection Method for Nonlinear Programming, I. Linear Constraints. J. Soc. Indust. Appl. Math., 1960, 8: 181-217.

[3] H.-Y. Kwei, F. Wu and Y.-L. Lai. Extension of a Variable Metric Algorithm to a Linearly Constrained Optimization Problem-A Variation of Goldfarb's Algorithm. In: K.B. Haley, Ed., OR'78 (NorthHolland, Amsterdam, 1979, 955-974.

[4] F. Wu. A Super-linear Convergent Gradient Projection Type Algorithm for Linearly Constrained Problems. European Journal of Operational Research, 1984, 16: 334-344.

[5] D.-Z. Du and J. Sun. A New Gradient Projection Method. Math. Numer. Sinica (Chinese Series), 1984, 6(4): 378-386.

[6] H.-Y. Kwei and D.-Z. Du. A Superlinearly Convergent Method to Linearly Constrained Optimization Problems under Degeneracy. Acta Math. Appl. Sinica, 1984, 1(1): 76-84.

[7] Q.-G. Zeng. Extension of Rosen's Gradient Projection Method and the Proof of Its Global Convergence. Acta Math. Appl. Sinica, 1991, 14(3): 312-322 (in Chinese).

[8] M.J.D. Powell. Some Global Convergence Properties of a Variable Metric Algorithm for Minimization without Exact Line Searches. SIAM-AMS Proceeding, Vol.9, 1976.

\section{Error Correction}

On Vol.13, No.4, p.414 of our journal, the title should be:

\section{EXISTENCE AND UNIQUENESS OF GLOBAL SOLUTION OF NONLINEAR SCHRÖDINGER EQUATIONS ON $R^{2}$}

